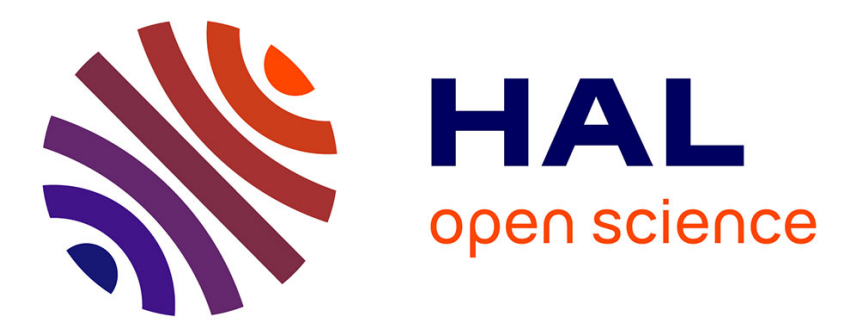

\title{
Error processing during online motor control depends on the response accuracy
}

Cécile' Galléa, Jozina de Graaf, Jean Pailhous, Mireille Bonnard

\section{To cite this version:}

Cécile' Galléa, Jozina de Graaf, Jean Pailhous, Mireille Bonnard. Error processing during online motor control depends on the response accuracy. Behavioural Brain Research, 2008, 193, pp.117 125. 10.1016/j.bbr.2008.05.014 . hal-01453855

\section{HAL Id: hal-01453855 \\ https://hal.science/hal-01453855}

Submitted on 22 Nov 2017

HAL is a multi-disciplinary open access archive for the deposit and dissemination of scientific research documents, whether they are published or not. The documents may come from teaching and research institutions in France or abroad, or from public or private research centers.
L'archive ouverte pluridisciplinaire HAL, est destinée au dépôt et à la diffusion de documents scientifiques de niveau recherche, publiés ou non, émanant des établissements d'enseignement et de recherche français ou étrangers, des laboratoires publics ou privés. 
Research report

\title{
Error processing during online motor control depends on the response accuracy
}

\author{
Cécile Galléa, Jozina Bernhardina de Graaf, Jean Pailhous, Mireille Bonnard* \\ Mediterranean Institute of Cognitive Neuroscience, UMR 6193, CNRS, Aix-Marseille University, 31 Chemin Joseph Aiguier, \\ 13402 Marseille, Cedex 20, France
}

\section{A R T I C L E I N F O}

\section{Article history:}

Received 19 October 2007

Received in revised form 29 April 2008

Accepted 2 May 2008

Available online 23 May 2008

\section{Keywords:}

fMRI

Human

Attention

ACC

SMA

\begin{abstract}
A B S T R A C T
We investigated which brain areas show error-related activity during online motor control while errors occur independently from decision making. During motor tasks, error is a deviation from accuracy or correctness. The effect of the accuracy level on error-related brain activity is unclear. Using functional Magnetic Resonance Imaging (fMRI), we investigated how error-related brain activity, especially in frontomedial wall areas, depended on motor accuracy (MA). Subjects performed a force tracking task with the thumb-index grip: to continuously follow a moving target on a monitor with a cursor which position was controlled by the force amount produced by the fingers. Task difficulty varied with changes in the cursor size (the smaller the cursor, the more difficult the task). We measured the motor accuracy (mean distance between the cursor center and the target) and the error amount (cursor out of the target). Errors were produced when motor accuracy was low and also when motor accuracy was high. For fMRI data processing, we defined a model based on both the error amount and the motor accuracy. The results showed that supplementary motor area (SMA) and dorsal anterior cingulate cortex (ACC) activation increased with error and task difficulty independent of the accuracy of motor control. Interestingly, activity in the rostral part of left ACC only increased with error when the motor accuracy was low, independently from task difficulty. These results suggest a clear functional dissociation between dorsal and rostral ACC in error processing which depends on the amount of attentional resources allocated to motor accuracy.
\end{abstract}

(c) 2008 Elsevier B.V. All rights reserved.

\section{Introduction}

An error refers to an act, an assertion, or a belief that unintentionally deviates from what is correct, right, or true ${ }^{1}$. Errors during an action elicit a specific pattern of brain activity [18] generated in the fronto-midline cortical areas [6]. Until now, most of the neuroimaging studies have focused on error-related brain activity that were based on decision making paradigms [6,10,14,29,32]. It is difficult to isolate error-related brain activity in those studies since the tasks examined induce both errors and response conflict (subjects make a choice between several possible responses among which one is correct). Furthermore in daily life, error can occur without making the wrong choice. During online motor control, perception and action are intimately coupled in a closed-loop system. In that case, errors can occur when the motor response is not adapted

\footnotetext{
* Corresponding author. Tel.: +33 491164 422; fax: +33 491164498 .

E-mail address: bonnard@incm.cnrs-mrs.fr (M. Bonnard).

1 Error (n.d.). The American Heritage $e^{\circledR}$ Dictionary of the English Language, fourth edition.
}

to environmental constraints, or when the task requirements are greater than the individual motor abilities. In this study, we have investigated which areas in the brain show error-related activity during online motor processes while errors occur independently from decision making.

The anterior cingulate cortex (ACC) shows event-related activity following not only incorrect trials $[17,48,50]$ but also correct trials when conflict monitoring increases in both cases [31]. Therefore, ACC activity has been attributed to control processes based on the co-activation of incompatible responses [4]. More generally, cognitive control theories report a role for the ACC in evaluating the gap between the current and desired response [4,5,33], or in allocating attentional resources $[19,35]$ when a discrepancy occurs. In order to distinguish between these alternative hypotheses, we carried out an online motor control study eliciting errors while the amount of resource allocation to the task varied. If the role of the ACC in error processes is linked to attentional resource allocation, then error-related activity will depend on the requirements of the online motor control task, the resource allocation being shared between the motor output requirements and error processing. If the role of the ACC is a generic monitor of error occurrence (i.e., when a gap 


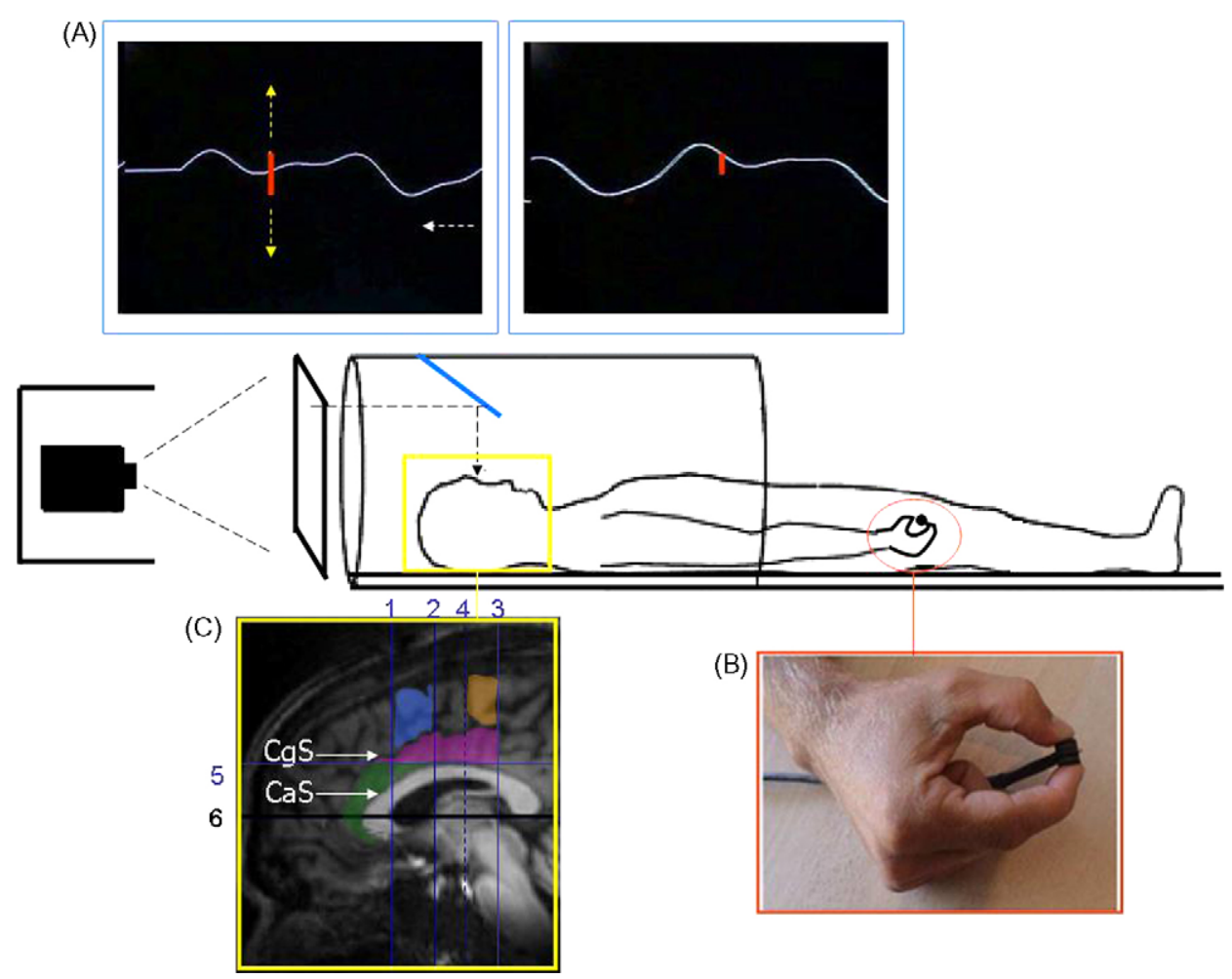

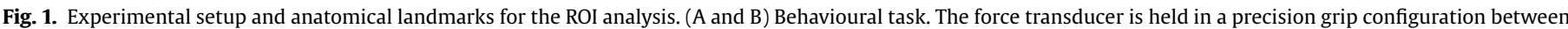

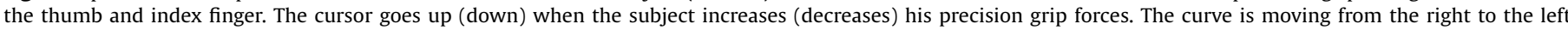

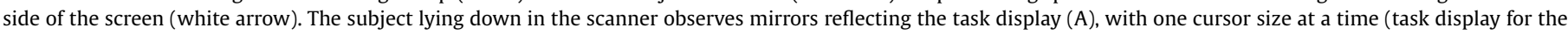

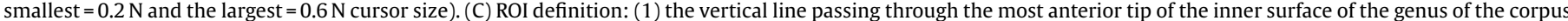

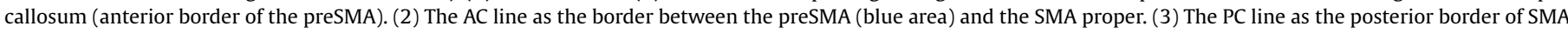

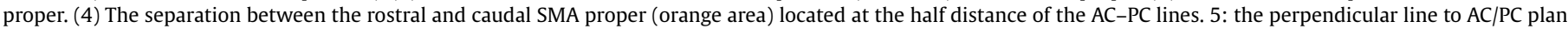
passing through the superior border of corpus callosum is the separation between the rostral (green) and ventral ACC (pink). CgS, cingulate sulcus, CaS, callosal sulcus.

between the current and the desired response occurs), then the ACC activity will be related to error independently of the motor requirements.

Medial-frontal cortex shows error-related activity during continuous motor tasks [30]. The error-related activity could be due to the ACC activation, although the supplementary motor area (SMA) could also be involved. Online motor control requires the ability to adapt the motor output relative to feedback about the performance, processes in which SMA is involved. The SMA proper is activated relative to regulation of the motor command to fit the task aim [54], according to the direct projections that SMA proper has on primary motor cortex (M1) and interneurons/motoneurons in the spinal cord $[13,20,39,40]$. The preSMA is the part of SMA with more cognitive properties with connections to the prefrontal cortex, ACC, but not directly to M1 [40,51]. The preSMA is activated in response to an increase in motor planning demands and during action monitoring $[17,40,54]$. When errors are correctable, they can be used to adapt the motor output to the task constraints [14,19,42]. Those compensatory behaviours require increased motor planning demands and motor regulations, processes in which the preSMA and the SMA proper play a role. Thus, both of the preSMA and the SMA proper could be involved in behavioural adaptation to error during the perception/action cycle.

To identify the differential involvement of ACC and SMA sub-regions when errors occur during online motor control, we designed an fMRI (functional Magnetic Resonance Imaging) study to measure the Blood Oxygenation Level Dependent (BOLD) response related to errors during an online visuomotor control task. The task involved various difficulty and motor accuracy (MA) levels. Subjects had to track an irregular target curve (moving from the right to left on a computer screen) while controlling the vertical position of a cursor (centrally on the computer screen) using force production. Defining a contrast that cancelled the effect of task difficulty, we were able to show that ACC activity was related to error in a way that depended on the amount of resources allocated to the accuracy of the motor output. In contrast, activity in the right preSMA and left SMA proper always depended on the error amount and task difficulty, meaning that activity in these regions was related to online adaptation to fit the motor output to the perceptual constraints independent of the amount of available resources allocated to motor accuracy.

\section{Material and methods}

\subsection{Subjects}

Twelve right-handed volunteers participated in this study ( 5 females and 7 males; mean age of all subjects $=26.7 \pm 4.8$ ). They were screened for fMRI compatibility, gave written, informed consent, and compensated for their participation. The protocol was approved by the ethics committee of the Public Assistance of Paris Hospitals (CCPPRB RBM 01-04).

\subsection{Behavioural task}

Subjects were instructed to track an irregular moving curve with cursor. The position of the cursor was controlled by a hard force transducer. The force transducer was held between the thumb and the index fingers of the right hand. The mean force that subjects produced to control the cursor position for each experimental condition was $5 \mathrm{~N}$. The applied forces were translated to the vertical position of the cursor, visible together with the curve on a screen (Fig. 1). Subjects were instructed to keep the cursor on the curve that moved from right to left on the screen. Task difficulty varied with 5 cursor sizes $(0.2,0.3,0.4,0.5$ and $0.6 \mathrm{~N})$, the physical cursor size corresponding to a safety margin of the force production to control the cursor relative to the target curve without error. The experiment contained 5 sessions (a 
session $=$ two $25 \mathrm{~s}$ trials of each cursor size randomly presented). The participants were trained for 1 hour 1 week before fMRI acquisition.

To verify that error occurrences increased with task difficulty, we measured the length of time the cursor was outside the curve for each trial (mean error occurrence per difficulty level). We measured the physical displacement of the cursor relative to the amount of force that subjects produced. The amount of error $(E)$ was defined as the sum of the error amplitude (distance in Newton between the center of the cursor and the target curve) at each error occurrence. The amount of errors refers to the sum of error events occurring intermittently during each trial. We also defined motor accuracy as a continuous variable to take into account the context of the motor task performance. MA refers to the mean distance between the cursor midline and the curve to track during each $25 \mathrm{~s}$ trial. We also calculated the coefficient of variation to verify the dispersion of the distribution of the cursor center position around the target curve (a higher variability meaning less accuracy).

It was considered higher motor accuracy to keep the location of the center of the cursor as close as possible to the target curve (concentric tracking) than to use the cursor width to track the curve (with a more important variability in the position of the cursor center relative to the curve). Errors could occur while the motor output accuracy was high, or while the precision of the motor output was sub-maximal. In order to separate these two cases, we categorized the individual trials by $E$ and MA values. Behavioural indicator $E$ and MA were integrated over each trial at the individual level. To analyze how error-related activity depended on motor accuracy, we classified high and low values relative to the medial for $E$ and MA. Each trial has been into four conditions: $\mathrm{E}^{+} \mathrm{MA}^{-}$(trials with high error amount and low motor accuracy), $\mathrm{E}^{-} \mathrm{MA}^{+}$(trials with low error amount and high motor accuracy), $\mathrm{E}^{+} \mathrm{MA}^{+}$ (trials with high error amount and high motor accuracy) and $\mathrm{E}^{-} \mathrm{MA}^{-}$(trials with low error amount and low motor accuracy).

Finally, we wanted to test whether errors were related to compensatory behaviour, i.e., whether the amount of motor regulations (force adaptation relative to performance feedback) increased with error amount. To address this, we measured the quantity of motor regulations for each trial, i.e., the power of the low frequencies in the force output produced by the subjects for each trial (recorded by the force transducer, quantified with a Fast Fourrier Transform). We chose to study the frequency range of $1.5-5 \mathrm{~Hz}$, the target curve presenting its main frequency peak at $1 \mathrm{~Hz}$. The chosen frequency range is a reasonable range given results of Vaillancourt et al. [49]. We analyzed the correlation between the error amount and the quantity of the motor regulation.

\section{3. fMRI data}

Imaging was carried out with a 3 Tesla whole-body Brucker Imager (MEDSPEC 30/80 AVANCE, Marseilles, France). High-resolution structural T1weighted images were acquired for anatomical co-registration ( $15 \mathrm{~min}$, voxel size $1 \mathrm{~mm} \times 0.75 \mathrm{~mm} \times 1.22 \mathrm{~mm}$ ). Functional images were acquired using a T2*weighted echo-planar sequence at 20 axial slices $(\mathrm{RT}=1.66 \mathrm{~s}$, interleaved acquisition, slice thickness $3 \mathrm{~mm}$, inter-slice gap $1 \mathrm{~mm}, 64 \times 64$ matrix of $3 \mathrm{~mm} \times 3 \mathrm{~mm}$ voxels). The slices, acquired parallel to the Anterior Commissure Posterior Commissure (AC-PC) plane, covered the upper brain and contained the frontal, parietal and the superior occipital lobes. Seven hundred ninety volumes of brain images were acquired per subject and submitted for analysis.

Statistical parametric mapping software (spm99 and spm2) was used for image processing and analysis (http://www.fil.ion.ucl.ac.uk/spm/). The functional images were interpolated in time to correct phase advance during volume acquisition, and realigned to the first image of each session. One subject was eliminated because of high head movement. The anatomical references and realigned functional images of all subjects were transformed to a common standard space using the Montreal Neurological Institute (MNI) template and smoothed at $10 \mathrm{~mm}$. The functional data were temporally filtered using a 120 s period high-pass filter and a Gaussian lowpass filter with a $4 \mathrm{~mm}$ Full Width and Half Maximum (FWHM). A General Linear Model (GLM) was defined for each subject relative to their behavioural performance $\left(\mathrm{E}^{+} \mathrm{MA}^{+}, \mathrm{E}^{+} \mathrm{MA}^{-}, \mathrm{E}^{-} \mathrm{MA}^{+}\right.$and $\left.\mathrm{E}^{-} \mathrm{MA}^{-}\right)$. Data were analyzed by modeling the four experimental conditions by a box-car convolved with the Hemodynamic Response Function (HRF) with $25 \mathrm{~s}$ durations.

We had a specific hypothesis about which region of the brain was involved in error processing. We, therefore, performed a Region Of Interest (ROI) analysis. Seven ROIs were defined based on individual anatomical data: left and right preSMA, left caudal SMA proper (cSMAp), left and right rostral ACC, and left and right caudal ACC (Fig. 1). Regarding the choice of left SMA proper, we were interested in the left sided structure for its involvement in controlling the right hand via corticospinal projections and M1 connection. The caudal part of SMA proper has a higher density in pyramidal cells than other SMA sub-regions [52] and thus, has more executive properties. ROI analyses were performed with the Marsbar SPM toolbox (http://marsbar.sourceforge.net/). We used the mean ROI activity to compute individual contrasts.

Concerning the ROI definition (Fig. 1C), the superior margin of the hemispheres was taken as the superior border of SMA. Inferiorly, SMA is bounded by the cingulate sulcus (CgS) [41]. The vertical line passing through the most anterior tip of the inner surface of the genus of the corpus callosum was taken as the anterior border of preSMA [8], the Anterior Commissural line as the boundary between preSMA and
SMA proper and the Posterior Commissural line as the posterior border of SMA proper [20]. The separation between rSMAp and cSMAp was located at the half distance of the AC-PC lines [52]. The superior border of ACC was the CgS [41], the lateral and inferior borders were the callosal sulcus. Finally, the perpendicular line to $\mathrm{AC} / \mathrm{PC}$ plan passing through the superior border of corpus callosum was considered as the separation between rostral and ventral ACC $[8,46]$.

We tested the differences in error amounts when the accuracy of the motor output is high $\left(\mathrm{E}^{+} \mathrm{MA}^{+}-\mathrm{E}^{-} \mathrm{MA}^{+}\right)$and differences in error amounts when the accuracy of the motor output is low $\left(\mathrm{E}^{+} \mathrm{MA}^{-}-\mathrm{E}^{-} \mathrm{MA^{- }}\right)$. We also performed contrasts testing the effect of the motor accuracy for equal error amount $\left(\mathrm{E}^{+} M \mathrm{~A}^{+}-\mathrm{E}^{+} M \mathrm{~A}^{-}\right)$ and $\left(\mathrm{E}^{-} M \mathrm{~A}^{+}-\mathrm{E}^{-} \mathrm{MA^{- }}\right)$. Results of the group analysis in random effect were obtained in terms of differences between regressor coefficients, that we will simply call "activation differences". For each ROI, the group results are shown with a chosen threshold at $p<0.05$ uncorrected, the analysis taking into account the mean activity of all the voxels taking part of the homogeneous anatomical ROI.

In a second step, we performed a whole brain analysis based on group results (random effect). Interaction contrasts were defined relative to our question of interest: are there any brain areas showing activation differences relative to the error amount when the accuracy of the motor output is high (i.e. $\left.\left(\mathrm{E}^{+} \mathrm{MA}^{+}-\mathrm{E}^{-} \mathrm{MA^{+ }}\right)>\left(\mathrm{E}^{+} \mathrm{MA}^{-}-\mathrm{E}^{-} \mathrm{MA}^{-}\right)\right)$or when the motor output accuracy is low (i.e. $\left.\left(\mathrm{E}^{+} \mathrm{MA}^{-}-\mathrm{E}^{-} \mathrm{MA}^{-}\right)>\left(\mathrm{E}^{+} \mathrm{MA}^{+}-\mathrm{E}^{-} \mathrm{MA}^{+}\right)\right)$? To answer this question, we defined the contrast $\left[\left(\mathrm{E}^{+} \mathrm{MA}^{-}-\mathrm{E}^{-} \mathrm{MA}^{-}\right)\right.$versus $\left.\left(\mathrm{E}^{+} \mathrm{MA}^{+}-\mathrm{E}^{-} \mathrm{MA}^{+}\right)\right]$. Because we were looking at small activation difference testing each voxel independently, we defined the threshold at $p<0.01$ uncorrected [16], since we were looking at very small differences. Finally, we performed a second level analysis on the whole brain using the individual error amount as a covariate for the contrast $\left(\mathrm{E}^{+} \mathrm{MA}^{-}-\mathrm{E}^{-} \mathrm{MA}^{-}\right)$using the threshold $p<0.01$ uncorrected.

\section{Results}

We aimed to study the effect of accuracy on error-related brain activity, independently from task difficulty. We analyzed the behavioural data relative to the amount of error $(E)$ and motor accuracy. Then, we defined the fMRI model based on the behavioural results of $E$ and MA with two properties: (1) the motor output did not fit the task requirements although the motor accuracy of the motor output could have been improved; (2) the motor output did not fit the task requirements but the accuracy of the motor output was already maximal. Thus, first, we tested the effect of identical error amount produced in two different contexts of motor accuracy. This first analysis was not independent to task difficulty. Second, we defined a contrast cancelling the effect of task difficulty to isolate the effect of motor accuracy on error-related brain activity.

\subsection{Behavioural data}

We calculated the mean error occurrence (length of time when the cursor was not on the target curve) over all subjects. The results showed that error occurrence increased with task difficulty (34.4\% of error for $0.2 \mathrm{~N} ; 17.3 \%$ for $0.3 \mathrm{~N} ; 8.3$ for $0.4 \mathrm{~N} ; 4.1 \%$ for the $0.5 \mathrm{~N} ; 2.3 \%$ for $0.6 \mathrm{~N}$ ). The amount of error is correlated with task difficulty to a greater extent than motor accuracy ( $E$ : Pearson coefficient $=0.65, p<0.05$; MA: Pearson coefficient $=-0.22, p<0.05$ ). This means that when task difficulty increased, subjects did not systematically control the motor output with more accuracy. For the $0.2 \mathrm{~N}$ cursor size (highest difficulty level), the mean duration of error occurrences across subjects was $372.21 \mathrm{~ms}( \pm 37.82 \mathrm{~ms})$ and the mean maximal duration of error occurrences was $1289 \mathrm{~ms}$ $( \pm 156.77 \mathrm{~ms})$. For the $0.6 \mathrm{~N}$ cursor size (lowest difficulty level), the mean duration across subjects was $310.80 \mathrm{~ms}( \pm 29.82 \mathrm{~ms})$ and the mean maximal duration of error occurrences was $918.9 \mathrm{~ms}$ $( \pm 150.55 \mathrm{~ms})$.

The results of the cursor size categorization as a function of the error amount $(E)$ and the motor accuracy of the behavioural output are shown in Fig. 2A. At least three different cursor sizes were always represented in the four conditions. The categorization of the individual trials per condition 
(A)
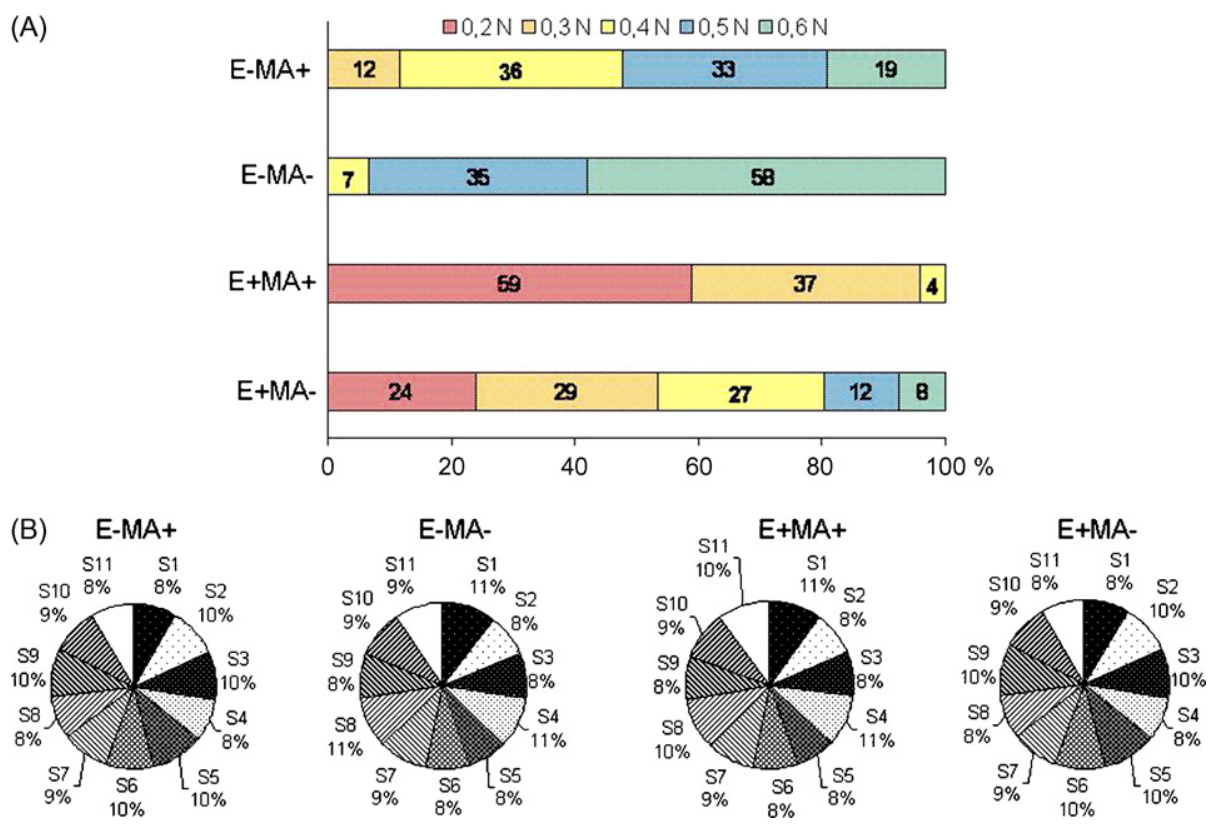

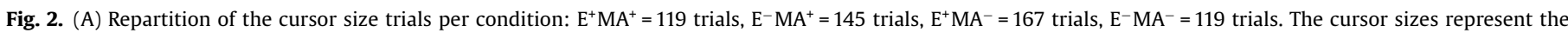

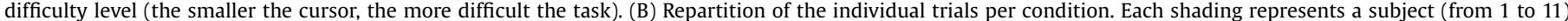

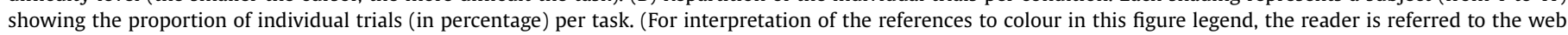
version of the article.)

showed that all the subjects were similarly represented in each condition (Fig. 2B). We calculated the mean difficulty level per condition: $\mathrm{E}^{+} \mathrm{MA}^{+}=0.2 \mathrm{~N}, \mathrm{E}^{-} \mathrm{MA}^{+}=0.4 \mathrm{~N}, \mathrm{E}^{+} \mathrm{MA}^{-}=0.4 \mathrm{~N}$ and $\mathrm{E}^{-} \mathrm{MA}^{-}=0.6 \mathrm{~N}$. In the fMRI part of the analysis (described in the next section), our contrasts of interest had equivalent differences in absolute difficulty level $\left(\left|\mathrm{E}^{+} \mathrm{MA}^{+}-\mathrm{E}^{-} \mathrm{MA}^{+}\right|=0.2 \mathrm{~N}\right)$ and $\left(\left|\mathrm{E}^{+} \mathrm{MA}^{-}-\mathrm{E}^{-} \mathrm{MA}^{-}\right|=0.2 \mathrm{~N}\right)$. Thus, in those 2 contrasts, we still test the effect of the error amount depending on MA and task difficulty increase. However, the interaction contrast $\left[\left(\mathrm{E}^{+} M \mathrm{~A}^{-}-\mathrm{E}^{-} \mathrm{MA^{- }}\right)\right.$ versus $\left.\left(E^{+} M A^{+}-E^{-} M A^{+}\right)\right]$tests the effect of the error amount cancelling the effect of task difficulty (equivalent in both contrast). Thus, the interaction contrast allows us to test the effect of MA on error-related brain activity, independently from task difficulty.

The $E$ and MA mean values are presented in Fig. $3 A$ and B. We calculated the correlation coefficient between $E$ and MA (Pearson coefficient $R=0.52, p<0.05$ ). This correlation was expected since when subjects made an error, they were less accurate. We performed an ANOVA on the $E$ and MA values obtained for each individual trial, sorted for the four conditions. There is a global effect of $E(F=174.32, p \leq 0.001)$ and MA $(F=95.33, p \leq 0.001)$. The post hoc test for multiple comparison (Bonferroni) showed significant difference in $E$ in comparing $\mathrm{E}^{+} \mathrm{MA}^{-}$and $\mathrm{E}^{-} \mathrm{MA}^{-}$as well as in comparing $\mathrm{E}^{+} \mathrm{MA}^{+}$and $\mathrm{E}^{-} \mathrm{MA}^{+}$. There was no significant difference of MA between $\mathrm{E}^{+} \mathrm{MA}^{-}$and $\mathrm{E}^{-} \mathrm{MA}^{-}$as well as in $\mathrm{E}^{+} \mathrm{MA}^{+}$and $\mathrm{E}^{-} \mathrm{MA}^{+}$(significant differences were observed in between low and high accuracy levels).We wanted to test the effect of $E$ on the quantity of voluntary force regulation in the motor output, on a range of $1.5-5 \mathrm{~Hz}$ (i.e., a frequency range related to online control based on feedback). We determined the mean power of the motor output in this range for each subject, for each condition. It appeared that the power significantly increased with the $E$ (Pearson coefficient $=0.34$, $p<0.05$ ), showing a correlation between the error amount and the voluntary force regulation (visible for the average values in Fig. 2B). In addition, the results of the ANOVA(E*MA, 2 levels for each factor) showed a significant effect of $E(F=6.743, p<0.05)$ and no signifi-
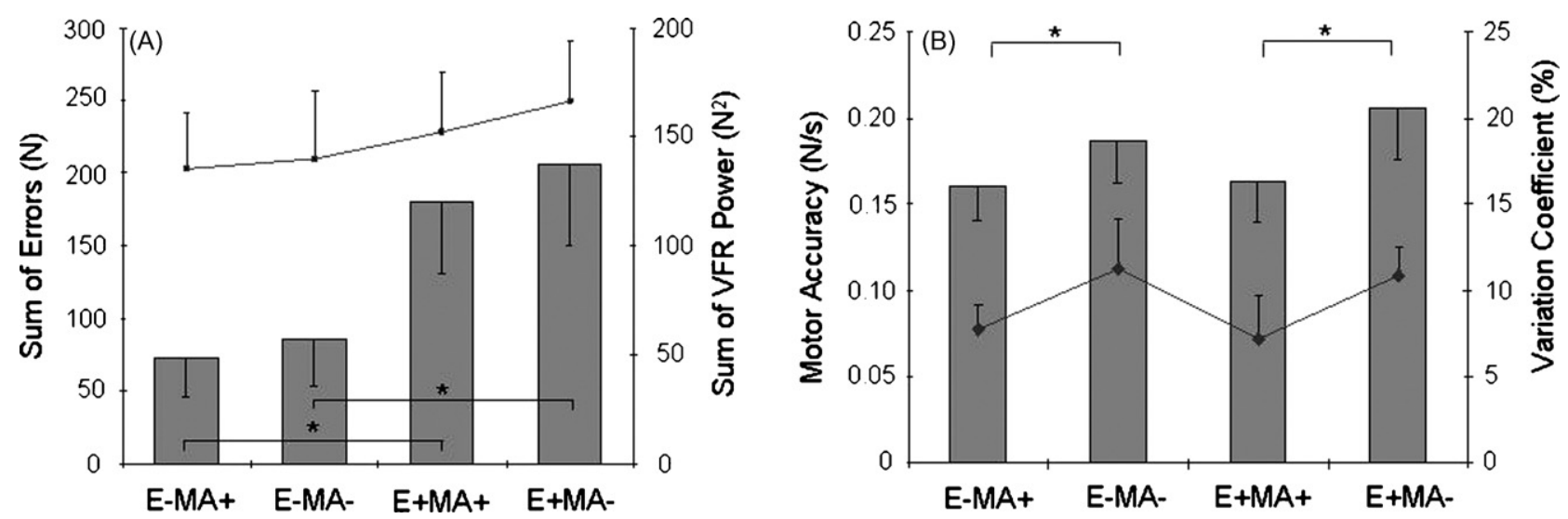

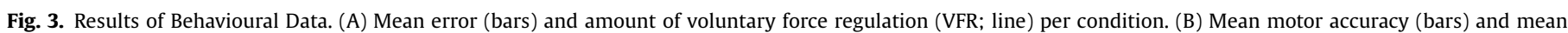
variation coefficient of the cursor center relative to the target curve (line) per condition. Significant differences between conditions are represented with black stars. 

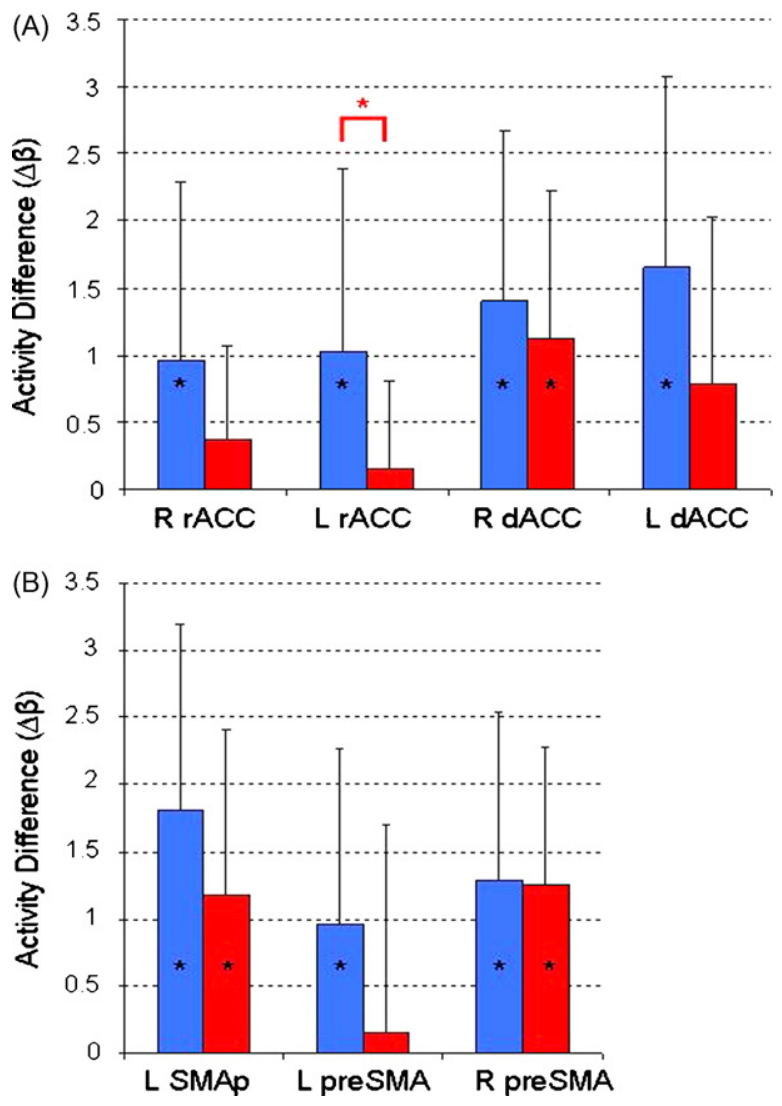

Fig. 4. Results of the ROI analysis. (A) ACC regions. (B) SMA regions. Beta Differences for the contrast $\left(\mathrm{E}^{+} \mathrm{MA}^{-}-\mathrm{E}^{-} \mathrm{MA}^{-}\right.$; red bars) and the contrast $\left(\mathrm{E}^{+} \mathrm{MA}^{+}-\mathrm{E}^{-} \mathrm{MA}^{+}\right.$; blue bars) are displayed for each ROI. Significant activity differences from zero are represented with a black asterisk. The red asterisk represents the significant difference in the left rostral ACC between contrasts $\left(\mathrm{E}^{+} \mathrm{MA}^{-}-\mathrm{E}^{-} \mathrm{MA}^{-}\right.$and $\mathrm{E}^{+} \mathrm{MA}^{+}-\mathrm{E}^{-} \mathrm{MA}^{+}$ contrasts) $(\Delta \beta=0.87, p<0.05)$. (For interpretation of the references to colour in this figure legend, the reader is referred to the web version of the article.)

cant effect of $\operatorname{MA}(F=1.088, p>0.3)$ on the amount of voluntary force regulation.

\section{2. fMRI data}

\subsubsection{ROI analysis}

We wanted to know whether activity in our ROIs increased with $E$ and whether this depended on MA. Results were obtained in terms of differences between beta regressor coefficients that we call "activation differences" (see Fig. 4). Measurements were made in bilateral preSMA, left caudal SMA proper, bilateral rostral and dorsal ACC. Bilateral rostral ACC error-related activity was statistically significant only when MA was low (right: $\mathrm{E}^{+} \mathrm{MA}^{-}-\mathrm{E}^{-} \mathrm{MA}-=0.96$, left: $\mathrm{E}^{+} \mathrm{MA}^{-}-\mathrm{E}^{-} \mathrm{MA}^{-}=1.03, p<0.05$ ). Errorrelated activity in right dorsal ACC, right preSMA and left caudal SMA proper was significant regardless of MA. Results of the group analysis in random effect were significant in the right preSMA $\left(\mathrm{E}^{+} \mathrm{MA}^{-}-\mathrm{E}^{-} \mathrm{MA}^{-}=1.29, \mathrm{E}^{+} \mathrm{MA}^{+}-\mathrm{E}^{-} \mathrm{MA}^{+}=1.26, p<0.005\right)$ and left caudal SMA proper $\left(\mathrm{E}^{+} \mathrm{MA}^{-}-\mathrm{E}^{-} \mathrm{MA}^{-}=1.82, \mathrm{E}^{+} \mathrm{MA}^{+}-\mathrm{E}^{-} \mathrm{MA}^{+}=1.79\right.$, $p<0.005)$. Those areas are activated relative to $E$ when task difficulty increased. The contrasts $\mathrm{E}^{+} \mathrm{MA}^{+}-\mathrm{E}^{+} \mathrm{MA}^{-}$and $\mathrm{E}^{-} \mathrm{MA}^{+}-\mathrm{E}^{-} \mathrm{MA}^{-}$ testing the effect of motor accuracy for similar error amounts showed no significant activity differences.

\subsubsection{Whole brain analysis}

We analyzed the interaction contrasts testing the effect of $E$ on the brain activity, depending on MA and indepen- dently from task difficulty (see Section 2). The first contrast $\left[\left(\mathrm{E}^{+} \mathrm{MA} \mathrm{A}^{-}-\mathrm{E}^{-} \mathrm{MA}^{-}\right)-\left(\mathrm{E}^{+} \mathrm{MA}^{+}-\mathrm{E}^{-} \mathrm{MA}^{+}\right)\right]$allowed us to isolate the brain areas showing more error-related activity when MA was sub-maximal compared to when MA is maximal. The second contrast $\left[\left(\mathrm{E}^{+} \mathrm{MA}^{+}-\mathrm{E}^{-} \mathrm{MA}^{+}\right)-\left(\mathrm{E}^{+} \mathrm{MA}^{-}-\mathrm{E}^{-} \mathrm{MA}^{-}\right)\right]$isolated the brain areas showing more error-related activity when MA is maximal compared when MA is sub-maximal. The first contrast showed error-related activity in left sided structures, especially in the rostral ACC (BA 32), putamen, caudate, inferior parietal cortex (BA 40) and primary sensorimotor cortex (SM1). The results are shown in Fig. 5A. The localization of clusters with the coordinates of their global max are shown in Table 1. The second contrast showed errorrelated activity in the right dorso-lateral prefrontal cortex only (cluster location: right medial-frontal gyrus; Talairach coordinates of the global maxima: 9, 57, 30; Number of voxel in the cluster: 11; $Z_{\max }=3.88$ ).

We performed a second level analysis for the contrast $\left(\mathrm{E}^{+} \mathrm{MA}^{-}-\mathrm{E}^{-} \mathrm{MA}^{-}\right.$) using the individual error amplitude (difference between the value of $E$ in the $\mathrm{E}^{+} \mathrm{MA}^{-}$condition and the value of $E$ in the $\mathrm{E}^{-} \mathrm{MA}^{-}$condition) as a covariate. At the behavioural level, we observed a larger mean difference in error $E$ in $\left(\mathrm{E}^{+} \mathrm{MA}^{-}-\mathrm{E}^{-} \mathrm{MA}^{-}\right)$

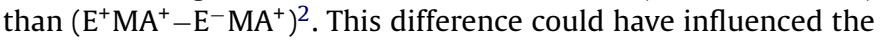
results obtained for the ROI analysis and the interaction contrast. Indeed, we observed systematically more difference of brain activity in the contrast showing slightly higher error amplitude at the behavioural level (Fig. 4). The second level analysis (using the error amplitude as a covariate) allowed us to verify the brain areas that showed an increase in BOLD signal that was correlated with the error amplitude. The results are shown in Fig. 5B and Table 2. None of the fronto-medial wall areas showed such a correlation. Only the left inferior parietal cortex (BA 40) showed an increase in activity

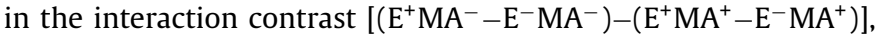
and a correlation with error amplitude. Other brain regions that showed a correlation with error amplitude were the right DLPFC, bilateral ventral premotor areas (vPM), left insula and right inferior posterior parietal cortex.

\section{Discussion}

We investigated the dependence of motor accuracy on errorrelated brain activity during the performance of a visuomotor task. Our ROI analysis showed that activity in the right dorsal ACC, right preSMA and left SMA proper was related to error rate independent of the motor accuracy. In a contrast cancelling the effect of task difficulty, we showed that the activity in the left rostral ACC, left posterior parietal cortex, left M1/S1, left caudate and putamen was related to errors for the lower motor accuracy level.

\subsection{Error, motor accuracy and task difficulty}

Studies about velocity/accuracy trade-off during pointing tasks $[15,54]$ showed that movement time increased with the motor accuracy and difficulty index, for a minimal error amount. The behavioural results of our study, imposing the tracking velocity, showed that error exponentially increases with task difficulty. Resource theories $[27,53]$ argue that performance trade-offs reflect the limitation of mental resources or processing capacity by the task being performed. Since the subjects of our study had to accurately

\footnotetext{
2 The amplitude difference is $14.22 \mathrm{~N}\left(\mathrm{E}^{+} \mathrm{MA}^{-}-\mathrm{E}^{-} \mathrm{MA}^{-}=205.83-85.3\right.$, $E^{+} \mathrm{MA}^{+}-\mathrm{E}^{-} \mathrm{MA}^{+}=179.76-73.45$, see Fig. $3 \mathrm{~A}$ for the values; $120.53-106.31=14.22$ ). Since the maximum of error occurrence over $25 \mathrm{~s}$ trial is $34 \%(=8.5 \mathrm{~s}$ of error occurrence), the maximal additional amplitude could be $\pm 1.6 \mathrm{~N}$ per second of error occurrence.
} 


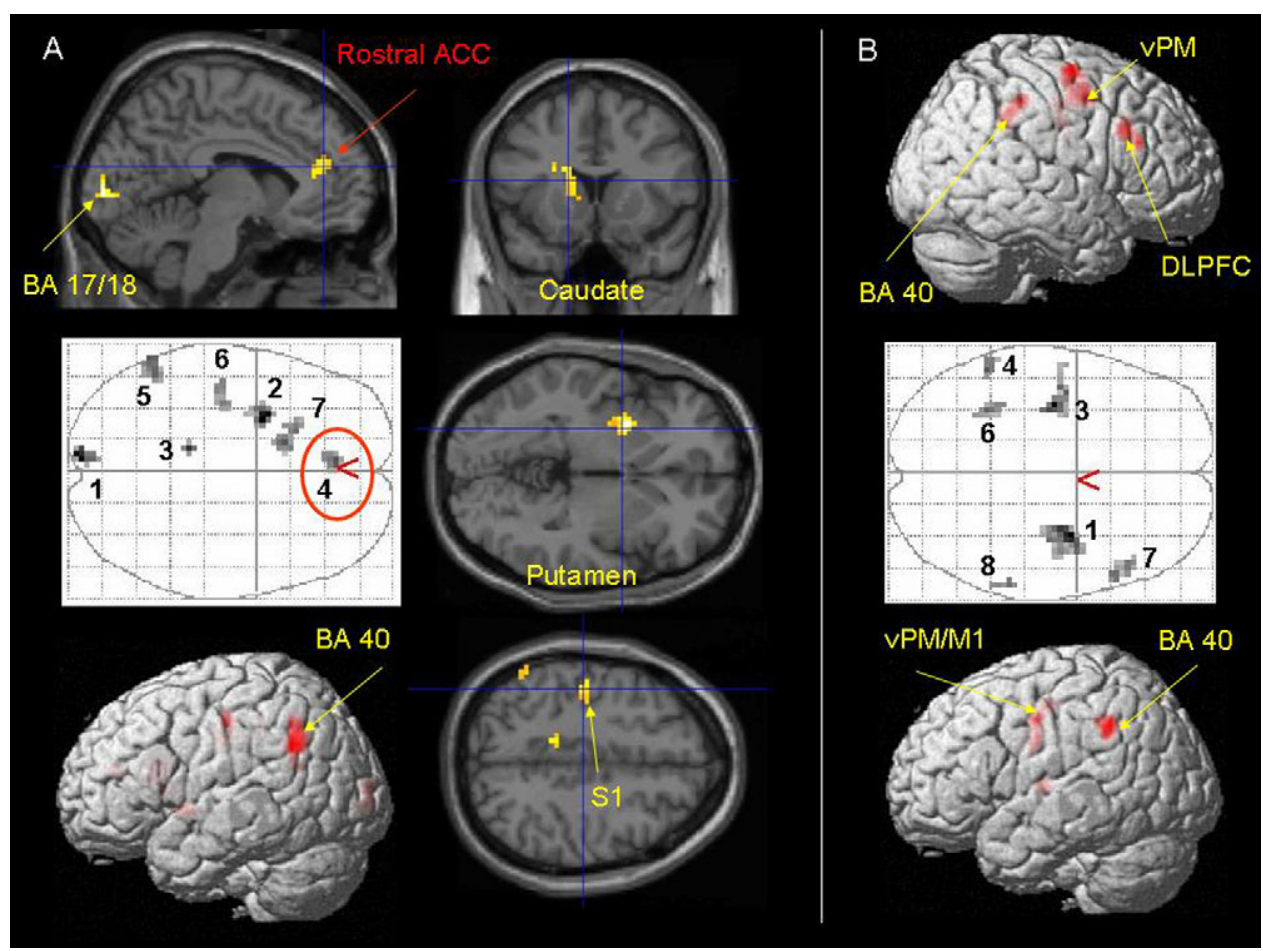

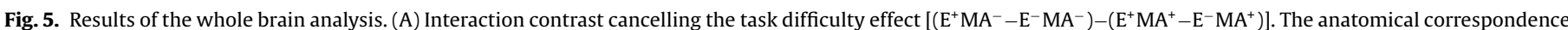

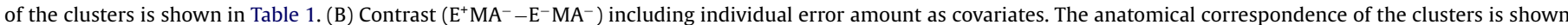
in Table 2 .

\section{Table 1}

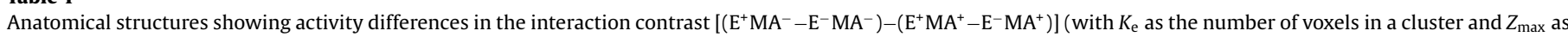
the value for each specified voxel above the threshold $p\left(Z_{\max }>u\right), p<0.01$ uncorrected)

\begin{tabular}{|c|c|c|c|c|}
\hline Cluster & Structures included in the cluster & Talairach coordinates & $K_{\mathrm{e}}$ & $Z_{\max }$ \\
\hline 1 & Left ligual gyrus, middle occipital gyrus (BA 17,18 ) & $-6-933$ & 27 & 3.58 \\
\hline 2 & Left putamen & $-273-3$ & 22 & 3.57 \\
\hline 3 & Left precuneus (BA 7) & $-12-3636$ & 20 & 3.33 \\
\hline 4 & Left medial-frontal gyrus (BA 32) & -64218 & 26 & 3.07 \\
\hline 5 & Left supramarginal gyrus (BA 40) & $-60-5733$ & 20 & 3.06 \\
\hline 6 & Left caudate & -151518 & 52 & 3.05 \\
\hline 7 & Left postcentral and precentral gyry (BA 1, 2, 3, 4) & $-36-1836$ & 27 & 2.81 \\
\hline
\end{tabular}

control their motor output and to monitor errors in parallel, information processing depended on the amount of available resources.

In Fitts's task, the accuracy was defined relative to the target width and depended on the difficulty index [15,54]. We measured motor accuracy as an active parameter taking into account the proximity of the cursor (controlled by the subjects) to the target. In some cases, we observed that the accuracy of the motor output was high (the cursor was closer to the target) at lower difficulty levels (the cursor was large). The subjects allocated their available resources to accurately control the motor output, decreasing the probability of error occurrence. Therefore, motor accuracy levels rather than task difficulty levels reflected the amount of resources allocated to online motor control.

\subsection{Role of ACC in error processing relative to the amount of available resources}

The interaction contrast showed that activity in the left rostral ACC activity is related to error independent of task difficulty. It has been reported that rostral ACC activity is specifically related to error monitoring [29,31]. However, our study of online motor control shows that the rostral ACC is not simply a monitor of error

Table 2

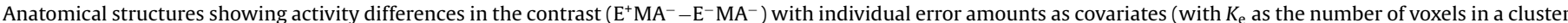
and $Z_{\max }$ as the value for each specified voxel above the threshold $p\left(Z_{\max }>u\right), p<0.01$ uncorrected)

\begin{tabular}{|c|c|c|c|c|}
\hline Cluster & Structures included in the cluster & Talairach coordinates & $K_{\mathrm{e}}$ & $Z_{\max }$ \\
\hline 1 & Right precentral gyrus (BA 6) & $33-388$ & 104 & 2.85 \\
\hline 2 & Left insula & $-36-126$ & 16 & 2.75 \\
\hline 3 & Left precentral gyrus (BA 4) & $-33-1551$ & 24 & 2.71 \\
\hline 4 & Left supramarginal gyrus (BA 40) & $-54-4839$ & 23 & 2.30 \\
\hline 5 & Left precentral gyrus (BA 4) & $-42-627$ & 18 & 2.28 \\
\hline 6 & Left postcentral gyrus (BA 2) & $-33-4836$ & 22 & 2.23 \\
\hline 7 & Right inferior frontal gyrus (BA 45/46) & 542133 & 26 & 2.19 \\
\hline 8 & Right supramarginal gyrus (BA 40) & $60-3645$ & 15 & 2.19 \\
\hline
\end{tabular}


occurrence. Indeed, activity in this area is not related to error when motor accuracy is high. Several studies reported that rostral ACC played a role in the evaluation of error response $[29,38,50]$ involving a comparison between the intended and the actual response [43]. The dorsal ACC is active after both error and feedback of the performance, also suggesting a role in performance evaluation $[5,38,45]$. Nevertheless, the activity differences in the ACC subregions of our subjects are not correlated with error amplitude, implying that their role in error/performance evaluation is nonspecific to the gap between the intended and the actual response. Our results show that the evaluative function in the rostral ACC depends on the amount of available resources. Thus, our current findings enhance the theories concerning the differential role of the dorsal and rostral ACC in error processing during online motor control.

Error-related activity in the rostral ACC depends on the amount of available resources required to accurately control the motor output. The rostral ACC is involved in the default brain network; activity in the rostral ACC decreases when resources are allocated to a demanding task [21]. This involvement is interpreted as performance optimization by allocating resources to task-necessary regions of the brain. Thus, a failure of performance optimization is supported by a relative activity increase (or less deactivation) in incorrect trials compared to baseline [38]. If such involvement can be isolated in the comparison of active states, the activity increase in the rostral ACC with errors when the motor accuracy is low can reflect a failure in performance optimization. Since rostral ACC involvement is not systematically observed when errors increase, it may be involved in resource allocation during online motor control independent of performance quality and compensatory behaviour following error [9,14,19,42].

It is known that the rostral part of the ACC plays a role in the appraisal of the affective or motivational significance of errors $[23,24,38]$. When subjects performed a concentric tracking, they kept the center of the cursor very close to the target with a continuous allocation of motivation and attentional focus. Thus, depending on the motor accuracy level, error can have different affective significance. If error-related activity in rostral ACC is due to an emotional appraisal, our results suggest that this mechanism is not automated and needs a certain amount of available resources. Therefore, whatever the role of this structure in more cognitive or emotional aspect of error, we show that rostral ACC role in error processing during online motor control is limited by the cognitive resources allocated to the ongoing task.

\subsection{Role of SMAproper, preSMA and $v P M$ in behavioural adaptation}

We observed an increase in the amount of voluntary force regulation with increasing error amplitude. This suggests that error information is used during the perception/action loop and that it induces compensatory behaviour. Right preSMA and left SMA proper activity is related to errors when task difficulty increases regardless of the amount of available resources. Receiving sensory input via the parietal cortex, the preSMA seems to identify task goals [26] and to participate in behavioural adaptation following errors [9]. During online motor control, this area is involved in the adaptation of the behavioural response to task constraints in order to reach the task goal [54]. In contrast, SMA proper participates in movement dynamic adaptation during motor execution [34], in motor adaptation to reduce errors [36], and more generally in determining the means to reach the goal [26]. Our results confirm SMA sub-region involvement when it is necessary to continuously match the actual performance with the task aim using feedback: the preSMA adapts the behavioural response to spatio- temporal constraints $[7,47]$ to reduce the discrepancy with the task goal, while SMAproper adapts the motor control to reduce errors.

Because the SMA proper and preSMA regions are equally involved when errors increase despite the motor accuracy, the interaction contrast does not show activity differences in those areas. Therefore, it is difficult to distinguish the role of the different SMA regions in error processing from their involvement in task difficulty. However, the preSMA is not differentially activated when task difficulty increases and when there is no difference in error rate [16,54]. It seems that SMA involvement depends on the ability to adapt and correct the motor response during online motor control when one has direct access to action consequences. Indeed, activity in preSMA and SMA proper is neither related to error and subsequent behavioural compensation during decision making [17-18] nor error during the execution of a discrete motor task [10]. Our findings enhance the existence of cognitive and executive processes in the SMA when errors are continuously relevant and require online adaptation of motor behaviour in response to perceptual feedback.

Activity in the bilateral PMv and left M1 but not left SMA proper is correlated to error amplitude. On the one hand, the result concerning SMA proper is unexpected since this structure has executive properties $[13,20,40]$ and the amount of motor regulations increased with error amplitude. On the other hand, left M1 activity increases with the need for motor regulation during visuomotor tracking tasks $[3,16]$ and lateral premotor areas are involved in visuomotor adaptation [55]. More precisely, error in execution that leads to adaptation in movement dynamics elicits increase in activity in the bilateral premotor areas compared to error that lead to a switch in the behavioural goal [12]. Thus, the PMv and SMA proper play different roles during online motor control. The lateral premotor areas seem to be involved in specific behavioural adaptation relative to error amplitude. The SMA proper contributes to a general behavioural adaptation as soon as a discrepancy between the motor performance and the task aim is detected regardless of its amplitude.

\subsection{Error processing during low motor accuracy}

BOLD signal in the left visual cortex, left posterior parietal cortex, left sensorimotor cortex, and left striatum increases with error when the motor accuracy is low, i.e., when there are more available resources for error processing. The posterior parietal cortex is involved in online error evaluation in response to sensory feedback $[2,11]$. We confirmed this idea by showing that the activation in this area is correlated with the perception of error amplitude. It seems that error information once evaluated by the frontal system (ACC) is then communicated to the posterior system (parietal cortex) for the adaptive modification of behaviour [30]. Our fMRI results did not provide sufficient time resolution to obtain precise delays of activation regarding to errors as events. Nevertheless, we show the involvement of the posterior parietal cortex, rostral ACC and left primary sensorimotor cortex when error increases during online motor control. The involvement of these areas is more important when there are available resources for error processing and behavioural adaptation. We show that reactive control using error feedback take place to ensure online motor adaptations.

Previous studies report that events appearing in the left or right visual field elicit activity in the visual areas of the contralateral hemisphere $[28,37,44]$. In our study, errors always occurred in the central field of view. Thus, error detection should involve bilateral visual areas, and the involvement of the left V1/V2 does not reflect error detection. In our study, the subjects could anticipate the shape of the curve which was visible on the right visual field $3.5 \mathrm{~s}$ before reaching the cursor in the central field of view. Because the antici- 
pation for motor control could only occur in the right visual field, we believe that the left lateralized visual activity reflects the existence of anticipation when resource availability allows it. The involvement of the putamen can also be related to anticipative control. Indeed, the striatum is involved in detecting context and using that context as a basis for predicting the outcome of actions [1,56]. In a context of low motor precision, when there are available resources for error processing, the striatum can provide a signal that leads to the programming and execution of behavioural adaptation to reduce errors through its cortical projections to motor structures $[1,22,25]$. During an online motor task, error processing depends on the amount of available resources possibly involving anticipation mechanisms.

\subsection{Conclusion}

During online motor control, the role of the rostral ACC in error processing is directly related to allocating available attentional resources when a discrepancy occurs between the actual performance and the task goal. In contrast, activation of the dorsal ACC and SMA are related to absolute action-goal discrepancy, regardless of the amount of available attentional resources. Error handling processes during a perception-action loop involve (1) the control of mismatch emergence signaling the need of behavioural adaptation that does not depend on the amount for available resources and (2) strategies for reducing the mismatch requiring a certain amount of attentional resources. The use of an online motor control paradigm eliciting errors allowed us to show error-related brain activity pattern independent to decision making. Further investigations in patients experiencing difficulty with accurate motor tasks like in Parkinson Disease or Dystonia should provide more information about brain (dys)function during online motor control and error processes without involving frontal tasks.

\section{Acknowledgements}

This study was supported be the Centre National de la Recherche Scientifique and the French program "Cognition and Information Processing". The authors would like to gratefully thank Professor J.M. Delarque who willingly assumes the medical responsibility for the experiment. We would like to thank Doctor Frederic Danion for his help in the behavioural part of the experiment. We are grateful to the reviewers for their suggestions and the NIH Fellows Editorial Board for their help and comments.

\section{References}

[1] Bar-Gad I, Havazelet-Heimer G, Goldberg JA, Ruppin E, Bergman H. Reinforcement-driven dimensionality reduction-a model for information processing in the basal ganglia. J Basic Clin Physiol Pharmacol 2000;11:305-20.

[2] Blakemore SJ, Frith CD, Wolpert DM. The cerebellum is involved in predicting the sensory consequences of action. Neuroreport 2001;12(11):1879-84.

[3] Bonnard M, Galléa C, de Graaf J, Pailhous J. Corticospinal control of the thumbindex grip depends on precision of force control: a TMS and fMRI study in humans. Eur J Neurosci 2007;25:872-80.

[4] Botvinick MM, Cohen JD, Carter CS. Conflict monitoring and anterior cingulate cortex: an update. Trends Cogn Sci 2004;8(12):46-539.

[5] Bush G, Vogt BA, Holmes J, Dale AM, Greve D, Jenike MA, Rosen BR. Dorsal anterior cingulate cortex: a role in reward-based decision making. Proc Natl Acad Sci USA 2002;99(1):523-8.

[6] Carter CS, Macdonald AM, Botvinick M, Ross LL, Stenger VA, Noll D, Cohen JD. Parsing executive processes: strategic vs. evaluative functions of the anterior cingulate cortex. Proc Natl Acad Sci USA 2000;97:1944-8.

[7] Coull JT. fMRI studies of temporal attention: allocating attention within, or towards, time. Brain Res Cogn Brain Res 2004;21:216-26.

[8] Crespo-Facorro B, Kim JJ, Andreasen NC, O'Leary DS, Wiser AK, Bailey JM, Harris G, Magnotta VA. Human frontal cortex: an MRI-based parcellation method. Neuroimage 1999;10:500-19.
[9] Debener S, Ullsperger M, Siegel M, Fiehler K, von Cramon DY, Engel AK. Trialby-trial coupling of concurrent electroencephalogram and functional magnetic resonance imaging identifies the dynamics of performance monitoring. J Neurosci 2005;25:11730-7.

[10] de Bruijn ER, Hulstijn W, Meulenbroek RG, Van Galen GP. Action monitoring in motor control: ERPs following selection and execution errors in a force production task. Psychophysiology 2003;40(5):786-95.

[11] Desmurget M, Grafton S. Forward modeling allows feedback control for fast reaching movements. Trends Cogn Sci 2000;4:423-31.

[12] Diedrichsen J, Grafton S, Albert N, Hazeltine E, Ivry RB. Goal-selection and movement-related conflict during bimanual reaching movements. Cereb Cortex 2006;16(12):1729-38.

[13] Dum RP, Strick PL. The origin of corticospinal projections from the premotor areas in the frontal lobe. J Neurosci 1991;11(3):667-89.

[14] Fiehler K, Ullsperger M, von Cramon DY. Electrophysiological correlates of error correction. Psychophysiology 2005;42(1):72-82.

[15] Fitts PM. The Information Capacity of the Human Motor System in Controlling the Amplitude of Movement. J Exp Psychol 1954;47:381-91.

[16] Galléa C, De Graaf JB, Bonnard M, Pailhous J. High level of dexterity: differential contributions of frontal and parietal areas. Neuroreport 2005;16(12):1271-4.

[17] Garavan H, Ross TJ, Murphy K, Roche RA, Stein EA. Dissociable executive functions in the dynamic control of behaviour: inhibition, error detection, and correction. Neuroimage 2002;17(4):1820-9.

[18] Garavan H, Ross TJ, Kaufman J, Stein EA. A midline dissociation between error-processing and response-conflict monitoring. Neuroimage 2003;20(2): 1132-9.

[19] Gehring WJ, Goss B, Coles MG, Meyer DE, Donchin E. A neural system for errordetection and compensation. Psychol Sci 1993;4:385-90.

[20] Geyer S, Matelli M, Luppino G, Zilles K. Functional neuroanatomy of the primate isocortical motor system. Anat Embryol (Berl) 2000;202:443-74.

[21] Greicius MD, Krasnow B, Reiss AL, Menon V. Functional connectivity in the resting brain: a network analysis of the default mode hypothesis. Proc Natl Acad Sci USA 2003;100(1):253-8.

[22] Gurney K, Prescott TJ, Redgrave P. A computational model of action selection in the basal ganglia. I. A new functional anatomy. Biol Cybern 2001;84:401-10.

[23] Hajcak G, Moser JS, Yeung N, Simons RF. On the ERN and the significance of errors. Psychophysiology 2005;42(2):151-60.

[24] Holroyd CB, Coles MG, Nieuwenhuis S. Medial prefrontal cortex and error potentials. Science 2002;296(5573):1601-10.

[25] Houk JC, Wise SP. Distributed modular architectures linking basal ganglia, cerebellum, and cerebral cortex: their role in planning and controlling action. Cereb Cortex 1995;5:95-110.

[26] Hoshi E, Tanji J. Differential roles of neuronal activity in the supplementary and presupplementary motor areas: from information retrieval to motor planning and execution. J Neurophysiol 2004;92(6):3482-99.

[27] Kahneman D. Attention and effort. Prentice-Hall: Englewood Cliffs; 1973.

[28] Kasai T, Morotomi T, Katayama J. Attending to a location in three-dimensional space modulates early ERPs. Cogn Brain Res 2003;17(2):273-85.

[29] Kiehl KA, Liddle PF, Hopfinger JB. Error processing and rostral anterior cingulate: an event-related fMRI study. Psychophysiology 2000;37(2):216-23.

[30] Krigolson OE, Holroyd CB. Evidence for hierarchical error processing in the human brain. Neuroscience 2006;137:13-7.

[31] Mathalon DH, Whitfield SL, Ford JM. Anatomy of an error: ERP and fMRI. Biol Psychol 2003;64(1-2):119-41.

[32] Menon V, Adleman NE, White CD, Glover GH, Reiss AL. Error related brain activation during a Go/NoGo response inhibition task. Hum Brain Mapp 2001;12(3):131-43.

[33] Milham MP, Banich MT, Claus ED, Cohen NJ. Practice-related effects demonstrate complementary roles of anterior cingulate and prefrontal cortices in attentional control. Neuroimage 2003;18(2):483-93.

[34] Padoa-Schioppa C, Li CS, Bizzi E. Neuronal activity in the supplementary motor area of monkeys adapting to a new dynamic environment. J Neurophysiol 2004;91(1):449-73.

[35] Pardo JV, Pardo PJ. The anterior cingulate cortex mediates processing selection in the Stroop attentional conflict paradigm. Proc Natl Acad Sci USA 1990;87(1):256-9.

[36] Paz R, Natan C, Boraud T, Bergman H, Vaadia E. Emerging patterns of neuronal responses in supplementary and primary motor areas during sensorimotor adaptation. J Neurosci 2005;25(47):10941-51.

[37] Pazo-Alvarez P, Amenedo E, Lorenzo-Lopez L. Effects of stimulus location on automatic detection of changes in motion direction in the human brain. Neurosci Lett 2004;371:111-6.

[38] Polli FE, Barton JJ, Cain MS, Thakkar KN, Rauch SL, Manoach DS. Rostral and dorsal anterior cingulate cortex make dissociable contributions during antisaccade error commission. Proc Natl Acad Sci USA 2005;102(43):15700-5.

[39] Porter R. The Kugelberg lecture. Brain mechanisms of voluntary motor commands-a review. Electroencephalogr Clin Neurophysiol 1990;76(4): 282-93.

[40] Rizzolatti G, Luppino G. The cortical motor system. Neuron 2001;31(6): 889-901.

[41] Roland PE, Zilles K. Functions and structures of the motor cortices in humans. Curr Opin Neurobiol 1996;6:773-81.

[42] Rodriguez-Fornells A, Kurzbuch AR, Munte TF. Time course of error detection and correction in humans: neurophysiological evidence. J Neurosci 2002;22(22):9990-6. 
[43] Scheffers MK, Coles MG. Performance monitoring in a confusing world: errorrelated brain activity, judgments of response accuracy, and types of errors. J Exp Psychol Hum Percept Perform 2000;26(1):51-141.

[44] Spironelli, Tagliabue M, Angrilli A. Asymmetrical hemispheric EEG activation evoked by stimulus position during the Simon task. Neurosci Lett 2006;399(3):215-9.

[45] Taylor SF, Martis B, Fitzgerald KD, Welsh RC, Abelson JL, Liberzon I, Himle JA, Gehring WJ. Medial frontal cortex activity and loss-related responses to errors. J Neurosci 2006;26(15):4063-370.

[46] Tzourio-Mazoyer N, Landeau B, Papathanassiou D, Crivello F, Etard O, Delcroix N, Mazoyer B, Joliot M. Automated anatomical labeling of activations in SPM using a macroscopic anatomical parcellation of the MNI MRI single-subject brain. Neuroimage 2002;15:273-89.

[47] Ullen F, Forssberg H, Ehrsson HH. Neural networks for the coordination of the hands in time. J Neurophysiol 2003;89(2):1126-35.

[48] Ullsperger M, Von Cramon DY. Subprocesses of performance monitoring: a dissociation of error processing and response competition revealed by event-related fMRI and ERPs. Neuroimage 2001;14:1387401.
[49] Vaillancourt DE, Slifkin AB, Newell KM. Visual control of isometric force in Parkinson's disease. Neuropsychologia 2001;39(13):1410-8.

[50] vanVeen V, Carter CS. The anterior cingulate as a conflict monitor: fMRI and ERP studies. Physiol Behav 2002;77(4-5):477-82.

[51] Vogt BA, Finch DM, Olson CR. Functional heterogeneity in cingulate cortex: the anterior executive and posterior evaluative regions. Cereb Cortex 1992;2(6):435-43.

[52] Vorobiev V, Govoni P, Rizzolatti G, Matelli M, Luppino G. Parcellation of human mesial area 6: cytoarchitectonic evidence for three separate areas. Eur J Neurosci 1998;10(6):2199-203.

[53] Wickens CD. Multiple resources and performance prediction. Theor Issues Ergonom Sci 2002;3:159-77.

[54] Winstein CJ, Grafton ST, Pohl PS. Motor task difficulty and brain activity: investigation of goal-directed reciprocal aiming using positron emission tomography. J Neurophysiol 1997;77(3):1581-94.

[55] Wise SP, Moody SL, Blomstrom KJ, Mitz AR. Changes in motor cortical activity during visuomotor adaptation. Exp Brain Res 1998;121(3):285-99.

[56] Zheng T, Wilson CJ. Corticostriatal combinatorics: The implications of corticostriatal axonal arborizations. J Neurophysiol 2002;87:1007-17. 